\title{
Applications of artificial intelligence in urological setting: a hopeful path to improved care
}

\author{
Sung-Jong Eun', Jayoung Kim², Khae Hawn Kim ${ }^{3, *}$ \\ 'Digital Health Industry Team, National IT Industry Promotion Agency, Jincheon, Korea \\ ${ }^{2}$ Departments of Surgery and Biomedical Sciences, Cedars-Sinai Medical Center, Los Angeles, CA, USA \\ ${ }^{3}$ Department of Urology, Chungnam National University Sejong Hospital, Chungnam National University School of Medicine, Sejong, Korea
}

Artificial intelligence (AI) has been introduced in urology research and practice. Application of Al leads to better accuracy of disease diagnosis and predictive model for monitoring of responses to medical treatments. This mini-review article aims to summarize current applications and development of $\mathrm{Al}$ in urology setting, in particular for diagnosis and treatment of urological diseases. This review will introduce that ma- chine learning algorithm-based models will enhance the prediction accuracy for various bladder diseases including interstitial cystitis, bladder cancer, and reproductive urology.

Keywords: Artificial intelligence, Machine learning, Urology, Urological diseases

\section{INTRODUCTION}

The advent of artificial intelligence (AI) marked one of the greatest advancements in technology. From smartphones to surgical robotics, AI has changed society in monumental ways. As AI technology continues to improve and advance, its applications in medicine will only expand even more (Mao and Vinson, 2018). AI in medicine can be divided into two classes: virtual and physical. Virtual AI includes informatics and systems-based learning, such as deep learning management of symptoms to guide treatment decisions. On the other hand, physical AI includes robots and nanotechnology for enhanced drug delivery (Hamet and Tremblay, 2017). Both branches of AI can contribute to incredible improvements in both patient care and healthcare management. These new tools and capabilities are particularly bound to make much-needed impacts in the field of urology.

Applications of AI would be beneficial across all relevant urological subdivisions, including benign urology and cancer. This reigns especially certain for analyzing massive amounts of pertinent data for diagnostics and prognostics. Machine learning (ML) is a discipline of AI that integrates statistics with algorithms to find relationships from data (O’Mahony et al., 2014). Such a tool can be applied to clinical data and creates robust risk models and redefines classifications of diseases. As medicine advances to an era of "big data" with an increasing amount of complex healthcare data, ML can be a powerful resource in navigating, elucidating, and applying information (Checcucci et al., 2020). This present review paper will provide an overview of the current state of $\mathrm{AI}$ in urology as well as future prospective and limitations.

\section{APPLICATION OF ARTIFICIAL INTELLIGENCE IN UROLOGICAL SETTING}

\section{Al in urine analysis}

Urine can provide a wealth of information for a variety of diseases and conditions, including interstitial cystitis and urolithiasis. Cytology of the urine can even detect high-grade malignancies of the urinary tract (McIntire et al., 2019). However, the lack of standardized screening and poor accuracy due to manual observation can lead to unreliable and variable results (McCroskey et al.,

\footnotetext{
${ }^{*}$ Corresponding author: Khae Hawn Kim (id https://orcid.org/0000-0002-7045-8004 Department of Urology, Chungnam National University Sejong Hospital, Chungnam National University School of Medicine, 20 Bodeum 7-ro, Sejong 30099, Korea

Email:kimcho99@cnuh.co.kr

Received: September 12, 2021 / Accepted: October 10, 2021
} tribution Non-Commercial License (https://creativecommons.org/licenses/by-nc/4.0/) which permits unrestricted non-commercial use, distribution, and reproduction in any medium, provided the original work is properly cited. 
2015). Noting this discrepancy and need, Sanghvi et al. (2019) successfully developed an AI algorithm capable of accurately analyzing urine samples for high-grade urothelial carcinoma. Urinalysis is also particularly important for addressing urinary tract infections (UTIs), the most common outpatient infections in the United States (Medina and Edgardo, 2019). Accurate and quick identification of the microbes underlying the infection is essential for providing the right antibiotics. However, culturing of the urine can take a prolonged period of time. A study developed a new strategy for urinalysis of UTI by integrating mass spectrometry with ML, which allowed for accurate bacterial identification in less than 4 hours (Florence et al., 2019).

\section{Al in benign urological conditions and diseases}

In addition to urinalysis, $\mathrm{AI}$ can integrate into how other benign urological conditions and diseases are treated. For instance, Kadlec et al. (2014) developed an artificial neural network (ANN) that predicted patient outcomes after endourologic interventions for kidney stones. A separate study by Aminsharifi et al. (2017) developed a different ANN-based model that predicted outcomes for patients after percutaneous nephrolithotomy. Beyond predicting outcomes, various groups have also used AI to support physicians in diagnostics and treatment decisions. One study by Längkvist et al. (2018) created a deep convolutional neural network to help differentiate kidney stones from phleboliths in computed tomography $(\mathrm{CT})$ scans.

\section{Big data-based ML application in urological cancers}

Big data-based ML is a subfield of AI, which involves the development and deployment of dynamic algorithms to analyze data and facilitate complex pattern recognition. The basic prediction models of ML include adaptive boosted trees (AdaBoost), gradient boosted trees, k-nearest neighbor, support vector machines (SVMs), bagged SVM, and random forest. In the field of healthcare, ML has been increasingly successfully applied to preventive medicine, image recognition, diagnosis, personalized medicine, and clinical decision-making. In predicting urological cancers, ML has many applications, such as assisting in diagnosis, judging the stage and grade, providing reliable prognosis, predicting the incidence of postoperative complications, and evaluating the responses in individual therapy.

When compared with traditional statistical methods, AI application showed significantly better accuracy, suggesting that AI might assist the decision-making process of urologists (Catto et al., 2003). Authors found that the predictive capacities of relapse accuracy using ANN or Neuro-fuzzy modeling were much better (88\% and 95\%, respectively) than traditional statistical methods (71\%-77\%). Xu et al. (2017) used a ML model to analyze the 3-dimensional bladder wall texture features based on CT/magnetic resonance imaging (MRI) images, which can accurately distinguish tumor and normal bladder wall tissue. This method can reduce the number of invasive examinations, such as cystoscopy and pathology. Kouznetsova et al. (2019) established a ML model to predict early and late stages bladder cancer (BC) by identifying metabolites that characterize different stages of $\mathrm{BC}$. Other groups have used a ML model to predict the BC stage and grade before operation combined with CT or MRI technology (Wang et al., 2019). Song et al. (2020) developed a computational model that can use population-based BC data to predict 10-year overall survival without considering tumor grade.

ML algorithms can create recurrence and survival prediction models based on imaging and surgical data to evaluate the recurrence and survival rates of patients following 1,3 , and 5 years after cystectomy (Hasnain et al., 2019). Klén et al. (2019) created a ML model to assess potential risk factors for postoperative and surgical related mortality by analyzing patients at high risk of early death after radical cystectomy. Congestive heart failure and chronic lung disease were added to previously known independent prognostic risk factors for early death after cystectomy. This method only used preoperative data and can be used before surgery. Computer evaluation was evaluated on radiology information extracted from CT images of BC patients, and established a ML model to evaluate whether patients are sensitive to chemotherapy. This method significantly improves the diagnostic accuracy, helps reduce unnecessary complications, improves quality of life, and reduces costs.

Although ML is widely used in BC, there are still some limitations, such as the difficulty in quantitative analysis of observed endpoints or the inapplicability of generalizability in other data sets. Therefore, we need further verification to improve its accuracy and versatility. In this study, we aimed to identify the potential predictive features to immunotherapy specific to $\mathrm{BC}$.

\section{Al in bladder diary}

To manage patients with voiding dysfunction, hospitals have used various tools for patient management assistance, such as voiding charts. The void chart is one of the methods for doctors to objectively monitor the subjective symptoms of patients with voiding dysfunction. Since the diagnosis or treatment proceeds after doctors objectively patients' symptoms, the void chart is the start- 
ing point of studies on voiding dysfunction, thereby being one of the most important diagnostic methods.

Many studies show that even if patients write the charts after being well educated, these data are inaccurate (Jarvis et al., 1980; Kim et al., 2014; Webb et al., 1992). There are many variables that can be monitored in the voiding charts, but it is clinically impossible to apply this method to all patients. In other words, there are many variables in voiding charts due to the issue of handwriting by patients with various abilities, so it is difficult to manage through accurate voiding charts. If accurate sensing technology based on $\mathrm{AI}$ technology and monitoring function to manage the voiding charts are implemented, systematic and efficient management of patient urination will be possible. If patients can carry their voiding charts like a watch or beeper and automatically record urination, it will be helpful in studying symptoms and mechanisms that have not been revealed in many patients.

Most of study based on AI, it proposes a technology to recognize the movement of urination by analyzing the data of acceleration signals and gyro signals collected from smart bands. Various methods and learning algorithms for motion recognition have been proposed. Most studies have used static algorithms such as ANNs (Karmonik et al., 2019; Kim et al., 2020; Nikkola et al., 2020; Prabhakar et al., 2019) and K-means clustering (Baser et al., 2020; Fraley and Raftery, 2002; Moon and Cho, 2021), or dynamic time warping (Powar and Chemmangat, 2019) in combination with algorithms. However, a time series algorithm should be used to predict or classify dynamically changing time series data. Typical time series algorithms include dynamic Bayesian networks (Kamalabad and Grzegorczyk, 2020), hidden Markov models (HMMs) (Sonnhammer et al., 1998), and recurrent neural networks (RNNs) (LeCun et al., 2015). The HMM and RNN methods have been widely used for time series data. However, HMMs are not appropriate for learning sequential data because each step is only influenced by the previous one. In concerned, it applied an RNN-based long short-term memory (Hochreiter and Schmidhuber,1997) method to process patient urination recognition. It aims to improve the recognition rate of the user's urination and efficiently solve the existing issues such as the problem of not applying the past data. Thus, it developing an analytical technique for high accuracy while solving the limitation of existing studies.

The presented recognition technology of the patients' urination is an extension of the existing pattern recognition technology based on signal processing. This technology is similar to the technology of recognizing specific motions in the smart home care service. The proposed technology also recognizes the signal pattern of urination and measures the frequency and time of urination to automatically record the urination information of the patients. This study based on AI aims to develop a technology for recognizing patients' urination by collecting and analyzing sensed movement (acceleration and tilt angle) information in the patients' smart bands. This development is expected to lead to the implementation of the enduser's urination management monitoring system (Eun et al., 2021). Fig. 1 shows an example of a urination management monitoring system.

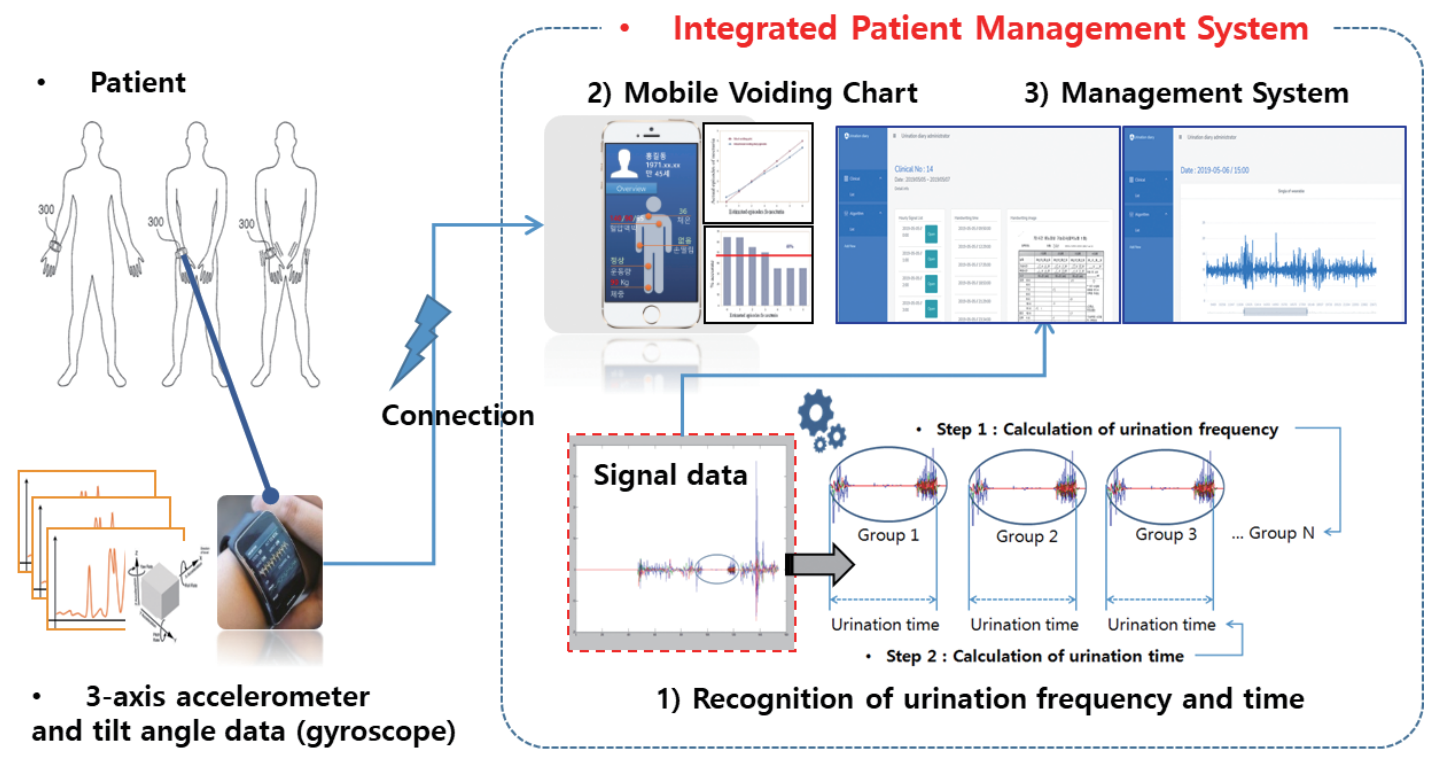

Fig. 1. The example of urination management monitoring system. 


\section{Al applications in reproductive urology}

The possibility of AI in medicine has been widely theorized over the past decades (Chung et al., 2019; Myung et al., 2019). Reproductive urology is a subfield where AI can contribute greatly because it has several limitations in the current predictive model and subjectivity within the field. Early AI application in reproductive urology focused on predicting semen parameters based on questionnaires identifying potential environmental factors and/or lifestyle influencing male fertility rates. AI has shown that genetic testing for anhydrosis has succeeded in predicting the number of patients most likely to need. With the recent development of image processing, automated sperm detection is a reality. The semen analysis, once a laboratory-only diagnostic test, has shifted to health consumer families with the emergence of AI. The prospects for AI in medicine are significant and have strong potential for $\mathrm{AI}$ in reproductive urology. Research identifying factors that may affect reproductive success by natural or assisted reproduction is of utmost importance to advance this field.

\section{CONCLUSIONS}

This mini-review article aims to summarize the current application and development of AI, especially in the urology environment for the diagnosis and treatment of urology diseases. The emergence of AI marked one of the biggest advances in technology. From smartphones to surgical robotics, AI has transformed society in a monumental way. As AI technology continues to develop and develop, its applications in the medical field will expand even further. $\mathrm{AI}$ in medicine can be divided into two types: virtual and physical. Virtual AI includes informatics and system-based learning, such as in-depth learning management of symptoms that guide treatment decisions. On the other hand, physical AI includes robots and nanotechnology to strengthen drug delivery. Both areas of AI can contribute to remarkable improvements in both patient care and medical care. These new tools and functions are bound to have a very necessary impact, especially on the urology field. The application of AI will be beneficial across all relevant urology fields, including benign urology and cancer. These tools can be applied to clinical data, creating a robust risk model and redefining disease classification. As medicine develops into an era of "big data" and "artificial intelligence" where the amount of complex medical data increases, it can be a powerful resource in the search, explanation, and application of information.

\section{REFERENCES}

Aminsharifi A, Irani D, Pooyesh S, Parvin H, Dehghani S, Yousofi K, Fazel E, Zibaie F. Artificial neural network system to predict the postoperative outcome of percutaneous nephrolithotomy. J Endourol 2017;31: 461-467.

Baser A, Eliaçı S, Baykam MM, Tan FU. Clinical manifestations of overactive bladder with migraine as a comorbidity: a prospective crosssectional study. Int Neurourol J 2020;24:375-381.

Catto JWF, Linkens DA, Abbod MF, Chen M, Burton JL, Feeley KM, Hamdy FC. Artificial intelligence in predicting bladder cancer outcome: a comparison of neuro-fuzzy modeling and artificial neural networks. Clin Cancer Res 2003;9:4172-4177.

Checcucci E, Autorino R, Cacciamani GE, Amparore D, Cillis SD, Piana A, Piazzolla P, Vezzetti E, Fiori C, Veneziano D, Tewari A, Dasgupta P, Hung A, Gill I, Porpiglia F. Artificial intelligence and neural networks in urology: current clinical applications. Minerva Urol Nefrol 2020;72: 49-57.

Chung KJ, Kim JY, Whangbo TK, Kim KH, The prospect of a new smart healthcare system: a wearable device-based complex structure of position detecting and location recognition system, Int Neurourol J 2019; 23:180-184

Eun SJ, Lee JY, Jung H, Kim KH. Personalized urination activity management based on an intelligent system using a wearable device. Int Neurourol J 2021;25:229-235.

Florence RD, Gotti C, Leclercq M, Hélie MC, Boissinot M, Arrey TN, Dauly C, Fournier F, Kelly I, Marcoux J, Julie BS, Bergeron MG, Droit A. Fast and accurate bacterial species identification in urine specimens using LC-MS/MS mass spectrometry and machine learning. Mol Cell Proteomics 2019;18:2492-2505.

Fraley C, Raftery AE. Model-based clustering, discriminant analysis, and density estimation. J Am Stat Assoc 2002;97:611-631.

Hamet P, Tremblay J. Artificial intelligence in medicine. Metabolism 2017; 69S:S36-S40.

Hasnain Z, Mason J, Gill K, Miranda G, Gill IS, Kuhn P, Newton PK. Machine learning models for predicting post-cystectomy recurrence and survival in bladder cancer patients. PLoS One 2019;14:e0210976.

Hochreiter S, Schmidhuber J. Long short-term memory. Neural Comput 1997;9:1735-1780.

Jarvis GJ, Hall S, Stamp S, Millar DR, Johnson A. An assessment of urodynamic examination in incontinent women. Br J Obstet Gynaecol 1980; 87:893-896

Kadlec AO, Ohlander S, Hotaling J, Hannick J, Niederberger C, Turk TM. Nonlinear logistic regression model for outcomes after endourologic procedures: a novel predictor. Urolithiasis 2014;42:323-327. 
Kamalabad MS, Grzegorczyk M. Non-homogeneous dynamic Bayesian networks with edge-wise sequentially coupled parameters. Bioinformatics 2020;36:1198-1207.

Karmonik C, Boone T, Khavari R. Data-driven machine-learning quantifies differences in the voiding initiation network in neurogenic voiding dysfunction in women with multiple sclerosis. Int Neurourol J 2019;23:195-204

Kim JK, Kim SJ, Park JM, Na YG, Kim KH. Past, present, and future in the study of neural control of the lower urinary tract. Int Neurourol J 2020; 24:191-199.

Kim SH, Oh SA, Oh SJ. Voiding diary might serve as a useful tool to understand differences between bladder pain syndrome/interstitial cystitis and overactive bladder. Int J Urol 2014;21:179-183.

Klén R, Salminen AP, Mahmoudian M, Syvänen KT, Elo LL, Boström PJ. Prediction of complication related death after radical cystectomy for bladder cancer with machine learning methodology. Scand J Urol 2019;53:325-331.

Kouznetsova VL, Kim E, Romm EL, Zhu A, Tsigelny IF. Recognition of early and late stages of bladder cancer using metabolites and machine learning. Metabolomics 2019;15:94.

Längkvist M, Jendeberg J, Thunberg P, Loutfi A, Lidén M. Computer aided detection of ureteral stones in thin slice computed tomography volumes using convolutional neural networks. Comput Biol Med 2018; 97:153-160.

LeCun Y, Bengio Y, Hinton G. Deep learning. Nature 2015;7553:436-444.

Mao S, Vinson V. Power couple: science and technology. Science 2018;316: 864-865.

McCroskey Z, Pambuccian SE, Kleitherms S, Antic T, Cohen MB, Barkan GA, Wojcik EM. Accuracy and interobserver variability of the cytologic diagnosis of low-grade urothelial carcinoma in instrumented urinary tract cytology specimens. Am J Clin Pathol 2015;144:902-908.

McIntire PJ, Khan R, Hussain H, Pambuccian SE, Wojcik EM, Barkan GA. Negative predictive value and sensitivity of urine cytology prior to implementation of the paris system for reporting urinary cytology. Cancer Cytopathol 2019;127:125-131.

Medina M, Edgardo CP. An introduction to the epidemiology and burden of urinary tract infections. Ther Adv Urol 2019;11:1756287219832172.

Moon JU, Cho KO. Current pharmacologic strategies for treatment of intractable epilepsy in children. Int Neurourol J 2021;25:S8-18.

Myung NV, Jung SY, Kim JY. Application of low-cost, easy-to-use, porta- ble biosensor systems for diagnosing bladder dysfunctions. Int Neurourol J 2019;23:86-87.

Nikkola J, Holm A, Seppänen M, Joutsi T, Rauhala E, Kaipia A. Repetitive transcranial magnetic stimulation for chronic prostatitis/chronic pelvic pain syndrome: a prospective pilot study. Int Neurourol J 2020;24:144149.

O’Mahony C, Jichi F, Pavlou M, Monserrat L, Anastasakis A, Rapezzi C, Biagini E, Gimeno JR, Limongelli G, McKenna WJ, Omar RZ, Elliott PM. A novel clinical risk prediction model for sudden cardiac death in hypertrophic cardiomyopathy (HCM risk-SCD). Eur Heart J 2014; 35:2010-2020.

Prabhakar AT, Ahmed ASI, Nair AV, Mathew V, Aaron S, Sivadasan A, Alexander M. Neural correlates of urinary retention in lateral medullary infarction. Int Neurourol J 2019;23:205-210.

Powar OS, Chemmangat K. Dynamic time warping for reducing the effect of force variation on myoelectric control of hand prostheses. J Electromyogr Kinesiol 2019;48:152-160

Sanghvi AB, Allen EZ, Callenberg KM, Pantanowitz L. Performance of an artificial intelligence algorithm for reporting urine cytopathology. Cancer Cytopathol 2019;127:658-666.

Song Q, Seigne JD, Schned AR, Kelsey KT, Karagas MR, Hassanpour S. A machine learning approach for long-term prognosis of bladder cancer based on clinical and molecular features. AMIA Jt Summits Transl Sci Proc 2020;2020:607-616.

Sonnhammer EL, Von HG, Krogh A. A hidden markov model for predicting transmembrane helices in protein sequences. Proc Int Conf Intell Syst Mol Biol 1998;6:175-182.

Wang H, Hu D, Yao H, Chen M, Li S, Chen H, Luo J, Feng Y, Guo Y. Radiomics analysis of multiparametric MRI for the preoperative evaluation of pathological grade in bladder cancer tumors. Eur Radiol 2019; 29:6182-6190.

Webb RJ, Fawcett PR, Neal DE. Electromyographic abnormalities in the urethral and anal sphincters of women with idiopathic retention of urine. Br J Urol 1992;70:22-25.

Xu X, Zhang X, Tian Q, Zhang G, Liu Y, Cui G, Meng J, Wu Y, Liu T, Yang $\mathrm{Z}, \mathrm{Lu} \mathrm{H}$. Three-dimensional texture features from intensity and highorder derivative maps for the discrimination between bladder tumors and wall tissues via MRI. Int J Comput Assist Radiol Surg 2017;12:645656. 